\title{
Automatic system for electroporation of adherent cells growing in standard multi-well plates*
}

\author{
Tomás García-Sánchez ${ }^{1}$, Maria Guitart ${ }^{2}$, Javier Rosell ${ }^{1}$, senior Member IEEE, Anna $\mathrm{M}^{\mathrm{a}}$ Gomez-Foix ${ }^{2,3}$ \\ and Ramón Bragós ${ }^{1}$, Member IEEE
}

\begin{abstract}
In this study an automatic system is presented to perform electroporation, also known as electropermeabilization, on adherent cells. It is an intention of this system to apply electric field pulses directly to cells growing in standard multiwell plates as a step forward to include this technique in standard laboratory protocols. An interdigitated microelectrode assembly constructed with Printed Circuit Board (PCB) is placed closely above the cell monolayer, and in order to avoid direct contact with cells, small micro-separators were included in the structure. Additionally, distribution of current density was modified by filling the gap between adjacent electrodes with a non conductive material as predicted by electric field simulations. This modification helps to concentrate the electric field intensity in the region where cells are present. The device was tested using $\mathrm{C2C12}$ cell line growing adhered in 24 multiwell plates and fluorescent labeled dextran FD20S as the molecule to be delivered. Successful transfection was observed with minimal invasiveness of the operation reducing the stress caused to cells.
\end{abstract}

\section{INTRODUCTION}

Electroporation, also called electropermeabilization, is a widely used technique to introduce foreign materials into the cells across the plasmatic membrane by means of pulsed high electric fields [1]. The main distinctive property of the technique is the fact that electroporation is based only on physical phenomena preventing the use of chemical agents. In the recent years there has been an increased interest in the use of the technique in clinical applications such as electrochemotherapy for cancer treatment or as a promising tool for gene therapy [2]. In vitro electroporation treatment has become a real alternative for some researchers, nevertheless its use is still far away to be applied as standard technique in usual laboratory protocols.

One of the reasons is that commercially available in vitro equipment is not designed to be used with standard cell culture plates. On the contrary, traditional electroporation is performed in specifically designed cuvettes where cells are suspended to apply electric field pulses [3]. Particularly, when adherent cells are electroporated a previous trypsinization process is necessary causing additional stress to the cells

\footnotetext{
*This study was supported by the project VALTEC09-1-0061 from Generalitat de Catalunya, ACC1Ó.

${ }^{1}$ T. García-Sánchez, J. Rosell and R. Bragós are are with the Electronic and Biomedical Instrumentation Group, Department of Electronics Engineering,Universitat Politecnica de Catalunya (UPC), Barcelona 08034, Spain. (phone: +34-934016777; e-mail: tomas.garcia.sanchez@upc.edu).

${ }^{2}$ M. Guitart and A.M. Gomez-Foix are with the Departament de Bioqumica i Biologia Molecular, IBUB, Universitat de Barcelona (UB), Barcelona.

${ }^{3}$ A.M. Gomez-Foix is also part of the CIBER de Diabetes y Enfermedades Metablicas (CIBERDEM).
}

that may affect both the efficiency and the invasiveness of the operation [4]. As explained in [1] there are several reasons to believe that in situ electroporation is more suitable for high efficacy transfection in adherent cells.

Some attempts to directly apply the electric field pulses to cells in state of adherence have been presented making use of thin film technologies to pattern microelectrodes structures on different surfaces [5]. In all of these approaches cells are attached onto the microelectrodes surface and not to a standard culture surface what may interfere its normal development. There are also reports of many single-cell devices introducing silicon fabrication technology [6]. Other setups treat cells directly attached to standard surfaces avoiding any contact with cells and focusing the effect of the treatment in a particular area [7][8]. However, all the devices described above are custom-built setups and are not suitable for their direct use in standard multi-well plates.

The aim of this paper is to present a new automated device to perform electroporation in situ to adherent cells growing on standard multi-well plates. Printed Circuit Board (PCB) cheap technology has been used in the fabrication of the microelectrode assembly allowing to have disposable electrodes with small enough dimensions. Such small dimensions allow to make use of low voltages to create high intensity electric fields, thus reducing the complexity and costs of the pulses generator required.

\section{MATERIALS AND METHODS}

\section{A. Principle of operation}

As pointed out in a previous study [9], cell attachment to the microelectrodes during electroporation creates a high permeability region located only around the area of contact between cells and electrodes. However, some molecules cannot precipitate to the bottom surface preventing them to pass across the membrane. Taking into account this fact and the principle of in situ application to cells growing attached on standard multi-well plates, we propose an automatic top positioning system capable of placing the electrodes momentarily on the bottom of the well-plates, just above the cell monolayer but not in contact with cells. A schematic representation of the top positioning principle and a detailed view of the electrode assembly is depicted in Figure 1.

\section{B. Electrode design and fabrication}

The vast majority of electroporation devices presented in previous studies make use of thin-film electrodes microfabricated with sputtering, lift-off and wet etching techniques 

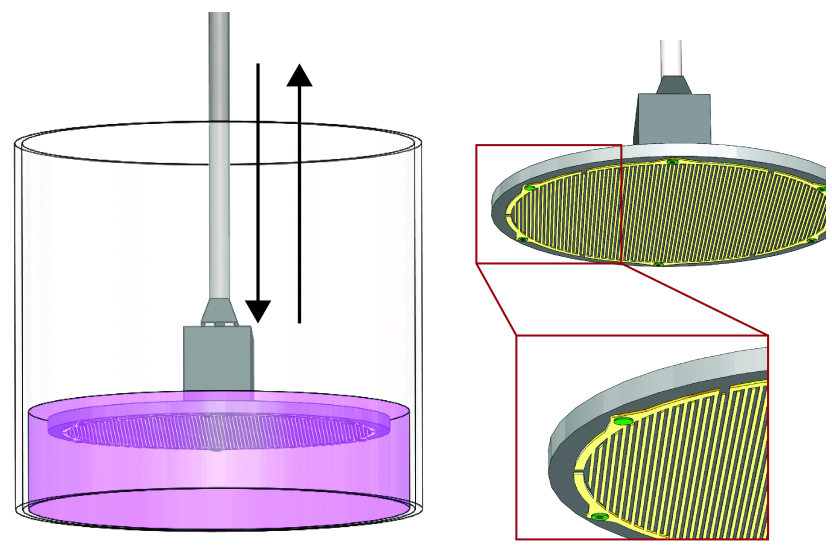

Fig. 1. Schematic representation of the top positioning principle and detailed view of the electrode assembly

[3][5]. These microfabrication processes enable utilization of really short interelectrode distances and a wide variety of materials but they have the disadvantage of increasing both the cost and complexity of the device. In the present study we propose to make use of standard printed circuit board (PCB) Class 7 bilayer technology according to high density integration (HDI) IPC regulation to manufacture our microelectrode assembly [10] (Lab Circuits S.A. 08460 Barcelona, Spain).

In the past few years, PCB technology has reached a resolution of tens of micrometers and some studies have been conducted to demonstrate the biocompatibility of a wide variety of materials used in PCB manufacturing processes in order to include this technology as a feasible option in the construction of lab-on-a-chip devices [11]. Additionally, this technology fulfills other specific requirements for the present case as the significant reduction in manufacturing costs. If one of our goals is to help electroporation to be introduced as a standard laboratory technique, it is desirable to supply low cost disposable devices. Other requirement is that connections with the electric field generator cannot be placed in the same plane where electrodes are patterned due to the top positioning principle described, this restriction is easily overcome with the standard PCB bilayer technology that allow to place the connector in the opposite face of the structure using metalized laser microvias to connect both sides, thus giving an easy top connectivity to the electrode assembly.

The microelectrodes where fabricated using $15 \mathrm{~mm}$ diameter discs made of FR4-135Tg as the substrate and a thin copper foil as the conductive layer. The chosen diameter is compatible with the dimensions of standard 24 multiwell plates. The design is based on a interdigitated electrode structure with each electrode array connected alternately to $+\mathrm{V}$ or $-\mathrm{V}$ terminals of the pulse generator. Some different designs were tested using different electrode width and spacing, the final dimensions used were $75 \mu \mathrm{m}$ for electrodes width and $150 \mu \mathrm{m}$ for the spacing. In order to avoid direct contact between the electrodes and the cell monolayer what could cause mechanical stress or damages to the cells, small micro-separations in the borders of the discs were patterned on top of the electrodes using a final photosensible epoxy layer with thickness of $10 \mu \mathrm{m}$. Thickness of cell monolayers is usually between $3 \mu \mathrm{m}$ and $8 \mu \mathrm{m}$ in most of cell lines attached to a surface [12] so a $10 \mu \mathrm{m}$ separation is high enough to avoid direct contact with the electrodes.

Due to the high thickness of the copper conductor foil (20 $\mu \mathrm{m})$ if compared with those of thin-film technologies, electric current density mainly concentrates in the lateral spacing between electrodes, furthermore if we take into account the relative distance between cells and electrodes, electric fields applied should be much greater than usual leading to electrolytic reactions that would irreversibly damage cell monolayer. As a solution we decided to fill the gap between electrodes with a non conductive photosensible epoxy resin in order to force the current density to concentrate in the surface of the structure.

A 2D simplification of the structure was simulated with commercial software (COMSOL Multiphysics 3.5, AC/DC module) with the exact size of the electrodes. Both numerical simulations include the FR4 substrate $(\sigma=0.004 \mathrm{~S} / \mathrm{m})$, copper electrodes $(\sigma=5.998 \mathrm{e} 7 \mathrm{~S} / \mathrm{m})$ and poration media $(\sigma=0.16$ $\mathrm{S} / \mathrm{m})$. Boundary conditions were set to fixed current port and ground alternatively. Two cases were studied, in Fig. 2A spacing between electrodes is not filled with any different material and in Fig. 2B the spacing is filled with photosensible epoxy resin $(\sigma=0.004 \mathrm{~S} / \mathrm{m})$. Results of the simulations demonstrate the increase in the current density in the area of interest by around twofold in the case where the spacing is filled with non conductive material what allows to lower the electric current intensities applied.

In addition, we plotted electric field cross-section values for the simulation in Fig. 2B. Electric fields created along two lines (marked in red in Fig. 2B) are shown in Fig. 3. One of them in the surface of the electrodes (dotted red line) and the other one (blue line) $15 \mu \mathrm{m}$ away from the surface where cells should be attached in the bottom of the wellplates. These plots help to demonstrate that considerably higher and irregular electric fields are presented in the border areas of the electrodes what could irreversibly damage cell -membranes near these areas. More uniform electric fields are created short distance away from the electrodes surface supporting the principle of operation of the device.

Finally, due to the non-biocompatibility of the copper $(\mathrm{Cu})$, once the electrode structure was patterned, a final Nickel(Ni)/Gold(Au) plating was deposited using Electroless Nickel Immersion Gold (ENIG) technique. The final result consists on a $\mathrm{Cu} / \mathrm{Ni} / \mathrm{Au}$ three-layered structure with thickness of $20 \mu \mathrm{m}, 0.7 \mu \mathrm{m}$ and $0.01 \mu \mathrm{m}$ respectively. This design is under patent process.

\section{Additional equipment}

Regarding the aim of standard laboratory use, an automatic system was designed to perform experiments in 24 multiwell plates inside of a sterile cabinet. This system is based 


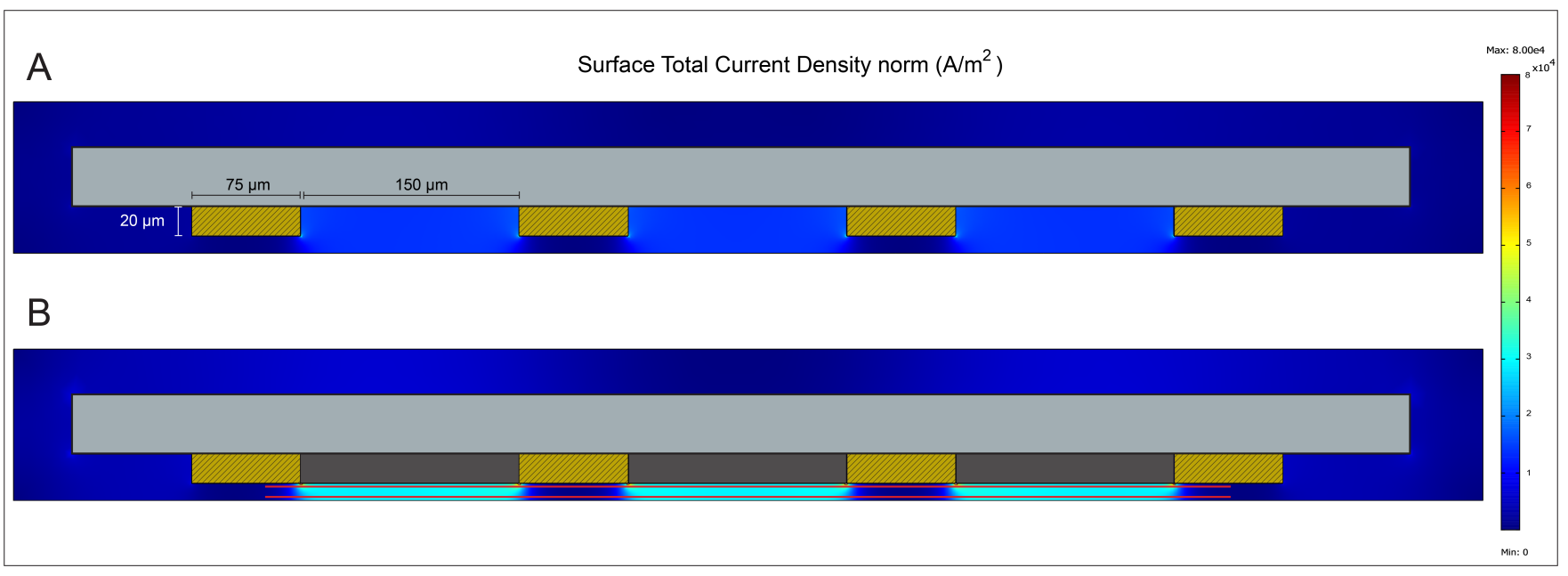

Fig. 2. Total current density simulations. Figure $2 \mathrm{~A}$ shows the distribution of current density when there is no different material between adjacent electrodes. In Figure 2B a non conductive material is placed between the electrodes. An increase in the current density is shown in the case of Figure $2 \mathrm{~B}$. Additionally in Figure 2B two red lines are marked for cross section values plotted in Figure 3.

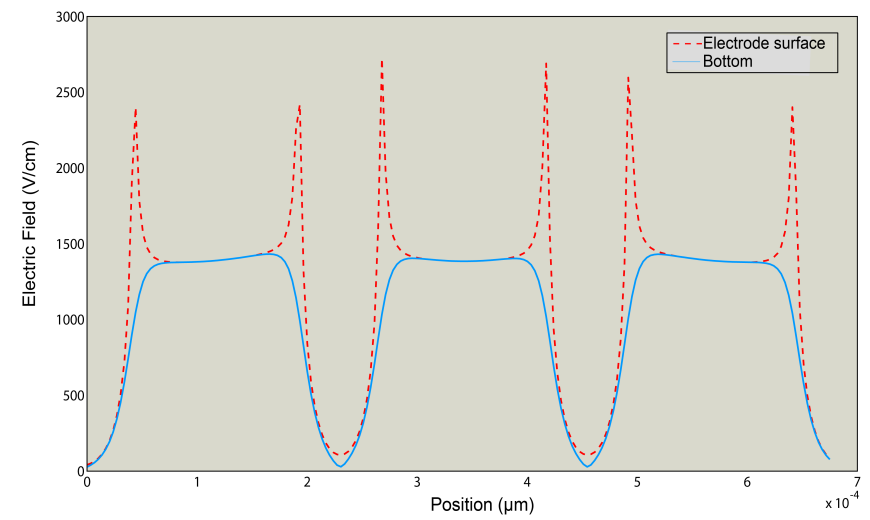

Fig. 3. Electric field intensity cross sectional plot. Values correspond with electric field level along the lines marked in Figure 2B. Dotted red line corresponds with the line marked in the surface of the electrodes, blue line corresponds with the line placed $15 \mu \mathrm{m}$ away from the surface.

on a self constructed $\mathrm{x}-\mathrm{y}$ positioning table where the multiwell is placed, and a $\mathrm{z}$ linear displacement posittioner where electrodes are mounted. Final part of the connection wires to the electrode assembly were introduced in a biomedical silicone tube whose flexibility allows the discs to be settled uniformly parallel to the bottom surface of the multi-well plates implying that electrodes will be completely parallel to the cell monolayer. The device makes use of stepper motors controlled by software Labview 2009 (National Instruments). The software also includes a user interface front panel where experimental electroporation conditions (current pulses intensity, duration, period and number of pulses) can be easily configured via RS-232 connection. Electric field pulse delivery was performed making use of a biphasic stimulator developed in our laboratory and first conceived for long-term contraction of cultured muscle cells. The stimulator generates bipolar pulses acting as a current fixed current source.

\section{Experimental protocol}

C2C12 mouse myogenic cell line was cultured as a monolayer in Dulbecco's Modified Eagle Medium (DMEM) supplemented with $10 \%$ fetal bovine serum and added with penicillin, streptomycin and fungizone. Low conductivity electroporation buffer (LCEB) was used in the experiments. LCEB consisted of $10 \mathrm{mM} \mathrm{Na} \mathrm{HPO}_{4} \mathrm{pH}$ (7.4), $1 \mathrm{mM}$ $\mathrm{MgCl}_{2}$ and $250 \mathrm{mM}$ sucrose. Conductivity of $1.6 \mathrm{mS} / \mathrm{cm}$ was measured experimentally using conductimeter GLP32 (Crison, Barcelona-Spain). When stated, $2.5 \mathrm{mg} / \mathrm{ml}$ fluorescein isothiocyanate-dextran average molecular weight 20000 Da (FD20S) (Sigma-Aldrich, Madrid, Spain) was added to the LCEB as fluorescence electropermeabilization probe.

C2C12 cell line was plated in 24-multi-well plates at a cell concentration of $35 \times 103 /$ well. The plates were cultured at $37^{\circ} \mathrm{C}$ in a humidified $5 \% \mathrm{CO}_{2}$ incubator for approximately $24 \mathrm{~h}$, to reach $50-60 \%$ confluence. Before the application of electric pulses, cells were rinsed with phosphate buffered saline (PBS) and then $250 \mu \mathrm{l}$ of LCEB were added to each well. The electrode assembly was sterilized with a $70 \%$ ethanol solution and then rinsed with sterile distilled water and finally immersed in LCEB before use. Immediately, the multi-well plate was placed in the automatic electroporation system. The electroporation conditions were fixed to four biphasic pulses, with $100 \mu \mathrm{s}$ separation between positive and negative part of the pulse, frequency repetition of $1 \mathrm{~Hz}$ and duration of $1 \mathrm{~ms}$ with different current intensities to create electric fields of $600,800,1000$ and $1200 \mathrm{~V} / \mathrm{cm}$. In control cells, no electric pulses were applied but the electrode was positioned above the cell monolayer for an equivalent period of time. After a period of 10 minutes following the application of pulses the electroporation buffer was removed and cells were rinsed twice with PBS and replaced with fresh culture medium and left for 2 hours in the incubator for complete resealing. Then, cells were examined under a Leica DMI 4000B inverted microscope for fluorescence, to 
detect FD20S, which has an excitation wavelength $485 \mathrm{~nm}$ and emission at $510 \mathrm{~nm}$. Images were taken with a digital camera Leica DFC 300x.

Viability was analyzed on the same treated cells after 24 hours of the electroporation procedure. Cells remaining adhered after this period were considered viable. Quick panoptic staining kit (Qumica clnica aplicada S.A., Amposta, Spain) was used to mark cells and then counted using free software ImageJ (MacBiophotonics).

\section{RESULTS AND DISCUSSION}

In the present study we tested the ability of the device to perform in situ electroporation of adherent cells attached to 24 multi-well plates. After a previous set of experiments to fix the optimal experimental conditions we decided to use four pulses with fixed duration of $1 \mathrm{~ms}$ varying the electric current intensities for different well plates. Experiments were duplicated in two different days under the same conditions and each condition repeated three times within each day.

Fig. 4 A shows the extent of permeabilization as a function of electric field intensity. Percentage of fluorescent cells was calculated dividing the number of marked cells by the total number of cells for each individual well plate. As expected, an increase in the electric field intensity applied led in an increase in the percentage of electroporated cells. For the best case $60 \%$ of cells on average resulted permeabilizated.
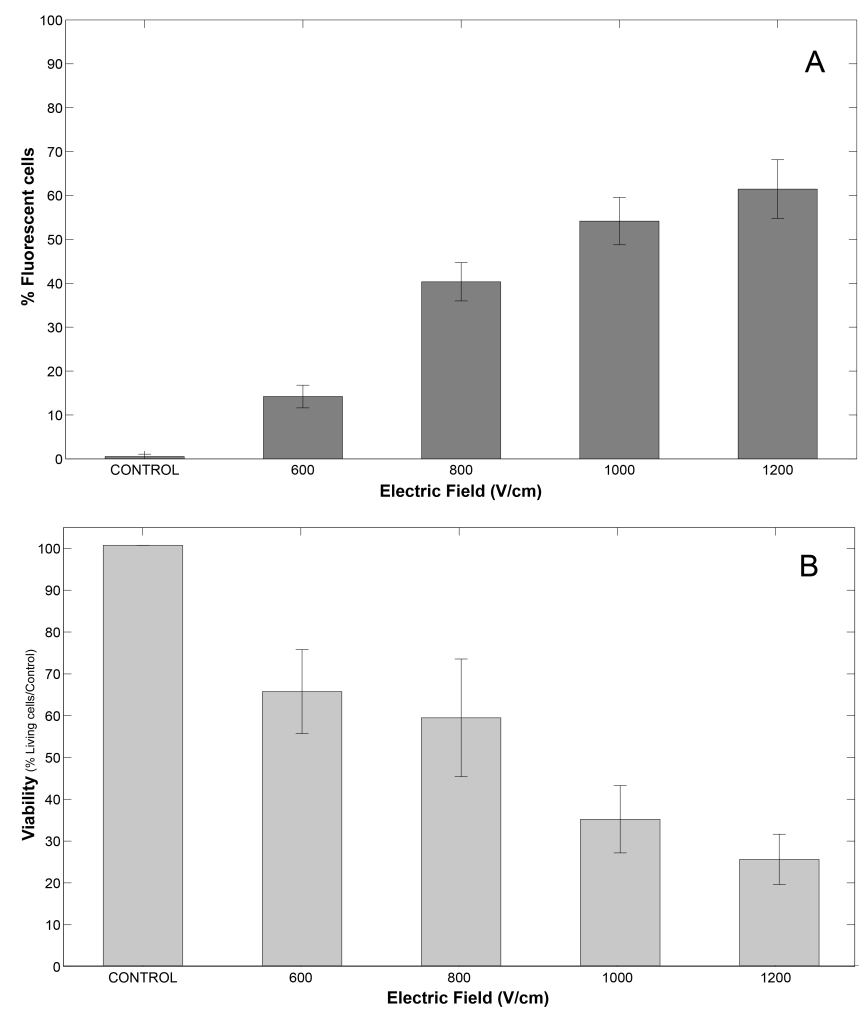

Fig. 4. Experimental results. Figure 4A shows the percentage of fluorescent cells for different electric fields as the number of cells marked with FD20S with respect to the total number of cells in each individual well. Figure 4B shows viability results for the same electric fields in Figure 4A 24 hours after application of treatment. Percentage was calculated as the number of attached cells divided by number of cells in control.
Fig. 4 B shows the number of viable cells after 24 hours of the electroporation procedure. The percentage of viability was calculated dividing the number of attached cells for each condition by the number of cells in control. Also as expected, viability decreased when electric fields were increased. For the lowest condition about $65 \%$ of cells remained viable after 24 hours.

The present results indicate effective electroporation of adhered $\mathrm{C} 2 \mathrm{C} 12$ cell line as stated by the uptake of FD20S into the cells cytoplasm with minimal invasiveness of the device. The treatment was applied directly to standard multiwell plates where cells had been initially grown avoiding additional stress to the cell culture by trypsinization or other post treatment steps. Although the reduction in manipulation of adherent cells should be translated in an increased viability comparing with other systems, our results show still excessive cell death. Even for the lowest electric field, viability is poor if compared with the extent of permeabilization for that condition. On the contrary, good results were observed in control cells where the microelectrode assembly was placed but no electric pulses were applied suggesting that indeed no mechanical damages were caused to the cell monolayer. We are currently working on how to improve viability results as well as the use of bioactive molecules such as DNA plasmids or siRNA in order to get high efficiency transfection.

\section{ACKNOWLEDGMENT}

We would like to thank Anna Orozco for her unconditional assistance.

\section{REFERENCES}

[1] D. C. Chang, B. M. Chassy, 1. A. Saunders and A. E. Sowers, Guide to Electroporation and Electrofusion, Academic Press, San Diego, 1992.

[2] Dev, S.B., et al., Medical applications of Electroporation. Plasma Science, IEEE Transactions on, 2000. 28(1): p. 206-223.

[3] Leda Raptis and Kevin L. Firth.Electrode Assemblies Used for Electroporation of Cultured Cells. Electroporation Protocols Preclinical and Clinical Gene Medicine. Humana Press 2008.p. 61.

[4] Q. Zheng, D.C. Chang, High-efficiency gene transfection by in situ electroporation of cultured cells, Biochem. Biophys. Acta 1088.

[5] Yu-Cheng, L. Electroporation microchips for gene transfection. in Emerging Information Technology Conference, 2005.

[6] Braeken, D., R. Huys, et al. Single-cell stimulation and electroporation using a novel $0.18 \mu \mathrm{m}$ CMOS chip with subcellular-sized electrodes. Engineering in Medicine and Biology Society (EMBC), 2010 Annual International Conference of the IEEE.

[7] De Vuyst, E., et al., In Situ Bipolar Electroporation for Localized Cell Loading with Reporter Dyes and Investigating Gap Junctional Coupling. Biophysical Journal, 2008. 94(2): p. 469-479.

[8] Olofsson, J., et al., Scanning Electroporation of Selected Areas of Adherent Cell Cultures. Analytical Chemistry, 2007. 79(12): p. 44104418.

[9] He, H., D.C. Chang, and Y.-K. Lee, Micro pulsed radio-frequency electroporation chips. Bioelectrochemistry, 2006. 68(1): p. 89-97.

[10] http://www.labcircuits.com/uploads/doc/Class7_eng.pdf

[11] Mazzuferi, M., et al., The biocompatibility of materials used in printed circuit board technologies with respect to primary neuronal and K562 cells. Biomaterials. 31(6): p. 1045-1054.

[12] Durante, M., et al., Thickness measurements on living cell monolayers by nuclear methods. Nuclear Instruments and Methods in Physics Research Section B: Beam Interactions with Materials and Atoms, 1993. 73(4): p. 543-549 